\title{
Aggressive T-LGL or indolent Hepatosplenic T-cell lymphoma: case with a difficult differential diagnosis
}

\author{
Prabhjot Kaur ${ }^{1}$
}

Received: 1 September 2015 / Accepted: 29 December 2015 /Published online: 15 January 2016

(C) Springer-Verlag Berlin Heidelberg 2016

T-cell large granular lymphocytic leukemia (T-LGL) is an indolent lymphoproliferative disorder characterized by persistent ( $>6$ months) increase in peripheral blood immunophenotypically distinct, clonal population of large granular lymphocytes, usually between $2-20 \times 10^{\wedge} 9 / \mathrm{L}$, without a clearly identifiable cause [1]. Hepatosplenic T-cell lymphoma (HSTL), on the other hand, is a uniformly aggressive disease with a fulminant systemic clinical presentation with massive hepatosplenomegaly, bone marrow involvement, thrombocytopenia and anemia, and B symptoms with survival counted in months (6-16 months) from the time of diagnosis [1]. More indolent variants are not recognized. This is a case with clinical presentation more compatible with T-large granular lymphocytic leukemia (T-LGL) and clinically followed as such, until it manifested clinically aggressively with B symptoms, splenomegaly, lymphadenopathy, and morphologically compatible with HSTL. This case continued to challenge us with the differential of a case of T-LGL with aggressive transformation or a HSTL with indolent presentation.

This is a case of a 65 -year-old lady, with a 10 -year history of Raynaud's disease, who presented with a mild viral syndrome associated with a slight leucopenia in September of 2007. In 2008, due to persistent cytopenia, a bone marrow biopsy was obtained. Her CBC at that time showed a wbc $1.4 \mathrm{k} / \mathrm{uL}$, with absolute neutropenia, $\mathrm{Hg} 13 \mathrm{gm} / \mathrm{dl}$, and platelets $137 \mathrm{~K} / \mathrm{uL}$. There were no B symptoms (fever, weight loss, night sweats). The peripheral smear failed to show an

Prabhjot Kaur

prabhjot.kaur@hitchcock.org

1 Department of Pathology, Dartmouth Hitchcock Medical Center and Geisel School of Medicine at Dartmouth, 1 Medical Center Drive, Lebanon, NH 03756, USA abnormal lymphocyte or large granular lymphocytic population. The bone marrow biopsy showed a small inter-trabecular nodular CD3-positive T-lymphocytic aggregate without significant interstitial T-cell infiltrates. Flow analysis showed an aberrant T-cell phenotype CD3+/CD8dim+/CD7 dim+/ CD56dim+/CD2+/CD16+/CD4-/CD5-/CD57- suspicious for a T-cell lymphoproliferative disorder. In 2009, the patient presented with pancytopenia, marked splenomegaly and mild hepatomegaly. The CBC showed wbc 1.7 (Neutrophil 340/ $\mathrm{uL}), \mathrm{Hg} 10.8$, and plt 80 . The bone marrow was hypercellular marrow $(70 \%)$ with decreased granulocytes, increased erythroid precursors, and normal-appearing megakaryocytes. There was an interstitial and moderate intrasinusoidal T-cell infiltrate containing small lymphocytes with round to slight irregular nuclear profiles and ample cytoplasm (HE, CD3+, TIA1+, Granzyme B equivocal negative) Fig. 1 (a-c). Flow analysis showed a similar aberrant phenotype $\mathrm{CD} 3+/$ CD8dim+/CD7dim+/ CD56dim+/ CD2+/CD16+/CD4-/ CD5-/CD57- and $\gamma \delta$ T-cell receptor-positive. Molecular studies were performed, and TCR beta and gamma gene rearrangements were seen. Cytogenetic studies showed a normal karyotype. FISH studies performed showed no chromosome 7 abnormalities. She was treated with G-CSF for her cytopenias. Unfortunately, this caused a splenic rupture (Fig. 1d). The spleen showed a diffuse red pulp disease with atrophic follicles. In June of 2010, she presented with B symptoms with fevers, night sweats, and a 10-pound weight loss and paraaortic and inguinal lymphadenopathy. There were persistent cytopenias: wbc 2.8 , rbc 3.18, plt $204 \mathrm{k}$. A lymph node biopsy was obtained. The lymph node architecture was effaced by a diffuse population of large transformed lymphocytes (Fig. 1e). By immunostains, they marked as CD3+, CD8+, TIA+ T-cell infiltrate with flow analysis showing a similar phenotype as reported earlier. A peripheral smear reviewed at this time showed numerous large granular lymphocytes (Fig. 1f). She 
Fig. 1 Bone marrow interstitial and intrasinusoidal T-cell infiltrates (a), CD3+ (b), TIA1+ (c), spleen (d), lymph node (e), and large granular lymphocytes (f)
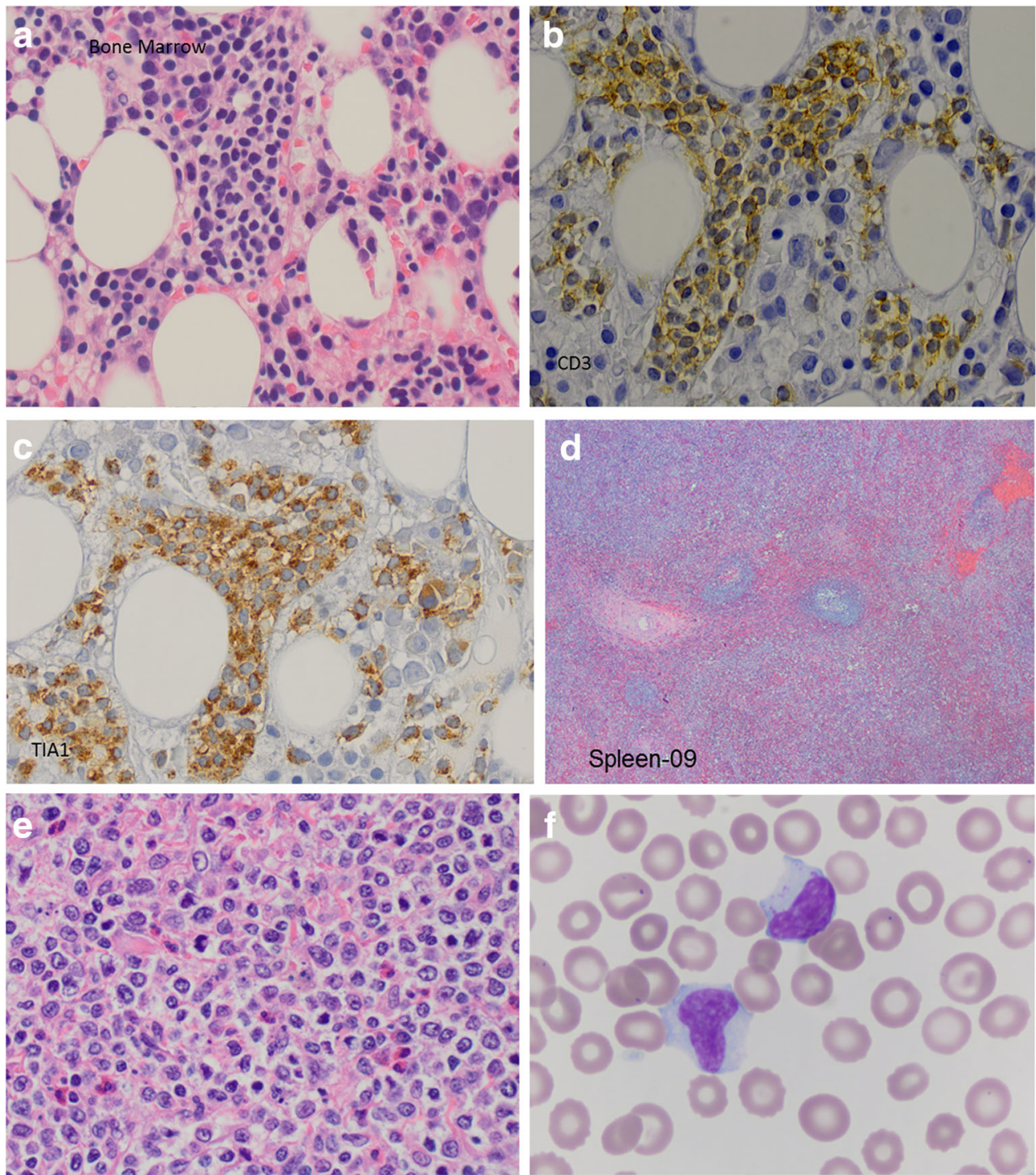

was treated with intensive chemotherapeutic regimen (RCHOP-14 $\times 6$ ). She however showed signs of progression post 6 cycles. Additional treatment included pralatrexate. By December 2010, she continued to have pancytopenia, effusions requiring transfusions and decided to pursue chemotherapy only to improve QOL without any additional aggressive transplant options.

On various fronts, this is an unusual case. Her indolent presentation was compatible with T-LGL. However, the morphologic features especially in the bone marrow from 2009 to 2010 and the splenic pathology point towards HSTL, that include lack of large granular lymphocytes in the peripheral blood and significant intrasinusoidal infiltrates in the BM. The spleen showed red pulp disease with atrophic follicles that is classically described in HSTL. Lymphadenopathy is not a feature of both and was seen in this case.

Each entity typically presents with a characteristic clinicopathologic profile. Briefly, T-LGL is an indolent lymphoproliferative disorder characterized by persistent ( $>6$ months) increase in the peripheral blood large granular lymphocytes, usually between $2-20 \times 10^{\wedge} 9 / \mathrm{L}$, without a clearly identifiable cause [1]. It is seen most commonly in the 4575 years age group with equal male to female ratio, presenting classically with neutropenia and/or anemia, lymphocytosis, splenomegaly, and rheumatoid arthritis. T-LGL is a leukemia of immunophenotypically distinct clonal T-cells, and morphologically, these infiltrates in the bone marrow can be extremely subtle. Stains for CD3, CD8 may highlight these infiltrates, and the diagnosis supported with appropriate flow and molecular studies [2, 3]. Majority of the cases show a CD3+/CD8+/ CD5 dim or negative/CD7 dim or neg/CD16+/-/CD56+/-/ CD57+ phenotype. NK-associated antigens CD94/NKG2, CD161 are expressed in at least $50 \%$ cases and clonal KIR/ no KIR seen in $50 \%$ cases [4]. They express TIA1, Granzyme $\mathrm{B}$ and $\mathrm{M}$, and/or perforin. T-LGL is usually of $\alpha \beta$ phenotype; however, $\gamma \delta$ subtype has also been reported with a 
comparable indolent clinical course [4]. Sandberg et al., summarize the clinical and pathologic findings on this subtype in a large series of cases. These patients present with cytopenia and may or may not present with hepatosplenomegaly, autoimmune disorders and other malignancies. These cases showed an indolent clinical course and were alive with disease after a follow-up of 3 years.

Hepatosplenic T-cell lymphoma (HSTL) is a rare, aggressive malignancy of cytotoxic T-cells with $\gamma \delta$ T-cell receptor type ([1] WHO). HSTL typically effects young adult males (35 years) and often has a fulminant systemic clinical presentation with massive hepatosplenomegaly, bone marrow involvement, thrombocytopenia and anemia, and B symptoms (fever, night sweats, weight loss). The neoplastic cells can be monotonous, with medium-sized cells. The pattern of infiltration is intrasinusoidal or a subtle interstitial pattern. With HSTL, disease progression increased numbers of large atypical cells with blast-like morphology may become more evident in the routine bone marrow aspirate and biopsy [5, 6]. The spleen shows red pulp involvement with atrophy of the white pulp. HSTL cells show inactive (immature) cytotoxic profile (i.e., positive for T-cell-restricted intracellular antigen(TIA-1) and negative for granzyme $\mathrm{B}$ and perforin) $[2,3,5,7]$ and $\mathrm{CD} 3+, \mathrm{CD} 4-, \mathrm{CD} 8-, \mathrm{CD} 5-$ and co-express NK-cell-associated antigens such as CD16 and CD56. HSTLs can be CD4-/CD8+. HSTL frequently expresses $\gamma \delta$ T-cell receptor heterodimers and less often $\alpha \beta$ T-cell receptor heterodimers $[5,7,8]$. Both have a similar clinical course. The cytogenetic abnormality seen in most cases is isochromosome $7 \mathrm{q}$ (either in isolation or associated with other clonal cytogenetic abnormalities including trisomy 8 and loss of chromosome Y) and can be identified by karyotypic analysis [5, 9, 10] or with targeted FISH analysis. Molecular studies show monoclonal TCRD, TCRB, and TCRG gene rearrangements by southern blot or PCR studies [7, 8]. The time to diagnosis is usually 60 days (15-180 days) and despite treatment with CHOP, platinum-cytarabine-based high-dose therapy [HDT] with SCT [10] survival is counted in months (6-16 months) from the time of diagnosis. The more classic cases of HSTL are reviewed elegantly by Vega $\mathrm{F}$ et al. [6].

As clinicapathologic entities, T-LGL is mostly indolent irrespective of $\alpha \beta$ or $\gamma \delta$ TCR expression. The spleen in TLGLs usually shows red pulp disease with hyperplastic follicles. There are rare aggressive variants of T-LGL reported in the literature [11-13] that present with significant lymphocytosis, hepatosplenomegaly, and B symptoms and have been mentioned in the WHO monogram. The pathology in aggressive variants shows red pulp disease with atrophic follicles [11], morphology similar to that seen in HSTL. Of note, spleen specimens from T-LGL are usually not biopsied, and the full morphologic spectrum may not be known. There is a single case report of $\alpha \beta$ T-LGL with subsequent transformation after 10 years of presentation [14]. Likewise, HSTL, an entity with a distinct clinicopathologic profile [15], is aggressive irrespective of $\alpha \beta$ or $\gamma \delta$ TCR expression. A single case report of an indolent HSTL has been reported with focus on treatment [16]. We considered this case as T-LGL with aggressive transformation. However, this case was considered an HSTL at the SH/EAHP workshop in 2012 at Lisbon, Portugal. Some opine that the factual data lacking specific genetic markers as seen in this case does not provide strong basis for definitive classification. We need more experience on HSTL that present with a more indolent clinical course or recognition of borderline cases that fail to fit a specified clinicopathologic entity. Hopefully, these will be addressed in forthcoming workshops and in the future WHO monograms.

\section{Compliance with ethical standards}

Funding This study did not require funding.

Conflict of interest The author declares no competing interests.

Ethical approval This article does not contain any studies with human participants or animals performed by the author.

\section{References}

1. WHO 2008: Gaulard P et al Hepatosplenic T cell lymphoma WHO 2008 292-293. WC Chan et al. T-cell large granular lymphocytic leukemia. WHO, 2008 Pages 272-275

2. Morice WG, Kurtin PJ, Tefferi A, Hanson CA (2002) Distinct bone marrow findings in T-cell granular lymphocytic leukemia revealed by paraffin section immunoperoxidase stains for CD8, TIA-1, and granzyme B. Blood 99(1):268-74

3. Morice WG, Kurtin PJ, Leibson PJ, Tefferi A, Hanson CA (2003) Demonstration of aberrant T-cell and natural killer-cell antigen expression in all cases of granular lymphocytic leukaemia. Br J Haematol 120(6):1026-36

4. Sandberg Y, van Dongen JJ, Miguel JF, Orfao A, Langerak AW (2006) TCRgammadelta + large granular lymphocyte leukemias reflect the spectrum of normal antigen-selected TCRgammadelta $+\mathrm{T}-$ cells. Leukemia 20(3):505-13

5. Cooke CB, Krenacs L, Stetler-Stevenson M, Greiner TC, Raffeld M, Kingma DW, Abruzzo L, Frantz C, Kaviani M, Jaffe ES (1996) Hepatosplenic T-cell lymphoma: A distinct clinicopathologic entity of cytotoxic gamma delta t-cell origin. Blood 88(11):4265-74

6. Vega F, Medeiros J, Gaulard P (2007) Hepatosplenic and other GD T cell lymphomas. Am J Clin Pathol 127:869-880

7. Belhadj K, Reyes F, Farcet JP, Tilly H, Bastard C, Angonin R, Deconinck E, Charlotte F, Leblond V, Labouyrie E, Lederlin P, Emile JF, Delmas-Marsalet B, Arnulf B, Zafrani ES, Gaulard P (2003) Hepatosplenic gamma/delta T-cell lymphoma is a rare clinicopathologic entity with poor outcome: report on a series of 21 patients. Blood 102(13):4261-9

8. Vega F, Medeiros LJ, Bueso-Ramos C, Jones D, Lai R, Luthra R, Abruzzo LV (2001) Hepatosplenic gamma/delta t-cell lymphoma in bone marrow. A sinusoidal neoplasm with blastic cytologic features. Am J Clin Pathol 116(3):410-9

9. Epling-Burnette PK, Painter JS, Chaurasia P, Bai F, Wei S, Djeu JY, Loughran TP Jr (2004) Dysregulated NK receptor expression in 
patients with lymphoproliferative disease of granular lymphocytes. Blood 103(9):3431-9

10. Macon WR M.D, Levy NB M.D, Kurtin PJ M.D, Cousar JB M.D (2001) Hepatosplenic [alpha][beta] T-Cell Lymphomas: a report of 14 cases and comparison with hepatosplenic [gamma][delta] T-cell lymphomas. Am J Surg Pathol 25(3):285-296

11. Gentile TC, Uner AH, Hutchison RE, Wright J, Ben-Ezra J, Russell EC, Loughran TP Jr (1994) CD3+, CD56+ aggressive variant of large granular lymphocyte leukemia. Blood 84(7):2315-21

12. Alekshun TJ, Tao J, Sokol L (2007) Aggressive T-cell large granular lymphocyte leukemia: a case report and review of the literature. Am J Hematol 82:481-485
13. Macon WR, Williams ME, Greer JP et al (1996) Natural killer-like T-cell lymphomas: aggressive lymphomas of T-large granular lymphocytes. Blood 87:1474-1483

14. Matutes E, Wotherspoon AC, Parker NE et al (2001) Transformation of T-cell large granular lymphocyte leukemia into a high-grade large T-cell lymphoma. Br J Haematol 115:801-806

15. Gaulard P, Belhadj K, Reyes F (2003) Gammadelta T-cell lymphomas. Semin Hematol 40(3):233-43

16. Bennett M, Matutes E, Gaulard P (2010) Hepatosplenic T cell lymphoma responsive to 2'-deoxycoformycin therapy. Am J Hematol 85(9):727-9 